\title{
Hongos micorrizicos arbusculares en el cultivo de plátano en viveros
}

\section{Arbuscular mycorrizical fungi in banana culture in nursery}

\section{Fungos micorrízicos arbusculares na cultura da banana em viveiros}

\author{
Jorge Vivas-Cedeño ${ }^{\mathrm{I}}$ \\ j.vivas02@hotmail.es \\ Yosbel Lazo-Roger II \\ ylazoroger81@gmail.com \\ Ignacio González-Ramírez III \\ igramirez3gmail.com \\ José Robles-García IV \\ jorobles55@hotmail.com
}

Recibido: 18 de enero de 2018 * Corregido: 23 de marzo de 2018 * Aceptado: 10 de junio de 2018

I. Ingeniero y Magister. Universidad Laica Eloy Alfaro de Manabí, Manta, Ecuador.

II. Ingeniero y Magister. Universidad Laica Eloy Alfaro de Manabí, Manta, Ecuador.

III. Ingeniero y PHD. Universidad Laica Eloy Alfaro de Manabí, Manta, Ecuador.

Iv. Ingeniero y Magister. Universidad Laica Eloy Alfaro de Manabí, Manta, Ecuador. 


\section{Resumen}

El desarrollo de nuevas tecnologías que beneficien la multiplicación y desarrollo inicial de plantas de plátano barraganete se ha convertido en la necesidad primordial para la renovación y establecimientos de nuevos cultivos en la región, por esto el objetivo de la investigación fue evaluar la influencia de la inoculación de cormos en Hongos Micorrizicos Arbusculares (HMA) en camas enraizadoras con dos sustratos en la obtención de plántulas de plátano barraganete; la investigación se realizó en El Carmen, provincia Manabí, se utilizaron tres dosis de HMA 15\%, 25\% y 35\% del peso del cormo, los dos sustratos fueron compuestos de arena + viruta de balsa, uno con y el otro sin humus; se evaluó la supervivencia, desarrollo morfológico, número de esporas en el sustrato y tasa de colonización en las raíces a los dos meses en vivero; El análisis estadístico mostró diferencias significativas entre los tratamientos en la supervivencia y desarrollo de las plántulas, las dosis de 25\% y 35\% de HMA en el sustrato con humus presentaron las respuestas más altas en el porcentaje de supervivencia, altura de planta, diámetro de pseudotallos, número de hojas y área foliar; estos tratamientos también presentaron los mejores resultados en el número de esporas en los sustratos y el mayor tasa de colonización de micorrizas en las raíces, las morfoespecies identificadas en el suelo en el análisis pertenecen al género Glomus y Acaulospora.

Palabras clave: micorrizas; arbusculares; esporas; barraganete; morfo especies.

\section{Abstract}

The development of new technologies that benefit the multiplication and initial development of banana plantations has become the main need for the renovation and establishment of new crops in the region, for this the objective of the research was to evaluate the influence of inoculation of corms in Mycorrhizal Arbuscular Fungi (AMF) in rooting beds with two substrates in the production of banana plantain seedlings; the investigation was carried out in El Carmen, Manabí province, three doses of $15 \%, 25 \%$ and $35 \%$ of the weight of the corm were used, the two substrates were composed of sand + balsa shavings, one with and the other without humus; Survival, morphological development, number of spores in the substrate and colonization rate in the roots at two months in the nursery were evaluated; The statistical analysis showed significant differences between the treatments in the survival and development of the seedlings, the doses of $25 \%$ and $35 \%$ of AMF in the substrate with humus presented the highest responses in the percentage of survival, height of plant, diameter of pseudostems, number of leaves and foliar area; these treatments also showed the best results in the 


\section{Hongos micorrizicos arbusculares en el cultivo de plátano en viveros}

number of spores in the substrates and the highest rate of mycorrhizal colonization in the roots, the morphospecies identified in the soil in the analysis belong to the genus Glomus and Acaulospora.

Keywords: mycorrhiza; arbuscular; spores; barraganete; morphospecies.

\section{Resumo}

O desenvolvimento de novas tecnologias que beneficiam a multiplicação eo desenvolvimento inicial das plantas de banana barraganete se tornou a principal necessidade de renovação e criação de novas culturas na região, de modo que o objetivo da pesquisa foi avaliar a influência da inoculação cormos em fungos micorriza arbusculares (HMA) em enraizadoras camas com dois substratos na obtenção de plântulas de bananeira barraganete; A pesquisa foi realizada, na província de Carmen Manabí, três doses de HMA utilizado $15 \%, 25 \%$ e $35 \%$ por peso de cormo, os dois substratos foram feitos de areia + jangada de chip, um com e outro sem húmus; Sobrevida, desenvolvimento morfológico, número de esporos no substrato e taxa de colonização nas raízes aos dois meses no viveiro foram avaliados; A análise estatística mostrou diferenças significativas entre os tratamentos sobre a sobrevivência e desenvolvimento da plântula, doses de $25 \%$ e $35 \%$ de HMA no substrato com húmus apresentaram as maiores respostas na percentagem de sobrevivência, altura da planta, diâmetro pseudostems, número de folhas e área foliar; estes tratamentos também tiveram os melhores resultados no número de esporos em substratos ea maior taxa de colonização micorrízica nas raízes, morfoespécie identificados na análise do solo e pertencem ao gênero Glomus Acaulospora.

Palavras chave: micorrizas; arbusculares; esporas; barraganete; morfo especies.

\section{Introducción}

El crecimiento económico del Ecuador tuvo un importante impulso debido a la producción de plátano en la región costa, gracias a que el país cuenta con excelentes condiciones climáticas, que permiten al cultivo mantener un desarrollo y producción constante durante el año, esto por las lluvias que mantienen la humedad necesaria para la actividad (PROECUADOR, 2015); a nivel nacional el plátano ocupa el cuarto puesto entre los cultivos de mayor superficie plantada con 110.110 hectáreas, y una producción de 610.413 toneladas al año, alcanzado una productividad 5,54 t.ha-1 (INEC, 2016).

Los problemas de productividad y limitaciones en el establecimiento de nuevas áreas de plátano, es la obtención de plántulas de calidad que garanticen una excelente plantación, entre los productores el método de siembra más utilizado es el directo, donde obtienen la semilla de otra planta y es colocada 
al lugar definitivo (Coto, 2009); sin embargo, este sistema presenta muchos problemas como el ataque de picudos, incidencias de nematodos y otras plagas, los cuales fuerzan al productor a utilizar productos químicos para desinfectar las semillas y reducir las pérdidas en el cultivo (Guerrero, 2011).

La aplicación de productos químicos es indispensable en este sistema de obtención de plántulas, para evitar las enfermedades o plagas en las plantas (Barea et al, 2018), pero en este método, el uso excesivo de estos productos puede ocasionar efectos negativos al ambiente; por eso se deben aplicar nuevas alternativas que disminuyan la utilización de químicos.

Una opción que permita disminuir el uso de químicos en las semillas de plantas, es la aplicación de biofertilizantes, especialmente los microorganismos del suelo, que benefician directamente a la planta en la absorción de nutrientes y la protección natural contra enfermedades y plagas (Rivera, et al, 2003), un grupo de estos microorganismos son las micorrizas, las cuales tienen una simbiosis abundante en la biosfera y en las raíces de las plantas optimizan sus funciones (Franco, 2008).

Las micorrizas son hongos que se desarrollan en el suelo y colonizan las raíces de las plantas sin ocasionar daños, al contrario, beneficia a las plantas mejorando su nutrición y generando resistencia a las plagas y enfermedades, además de volverla más tolerable a la sequía y salinidad (Barea et al, 2018), esta alternativa puede crear mayores beneficios económicos y ambientales, lo que garantizara una producción eficiente en el cultivo.

El uso y aplicación de micorrizas en la producción de plátano, tienen un mayor beneficio a una temprana edad de la planta, debido a que el desarrollo es más eficiente y brindan una mejor adaptabilidad al ambiente (Rivera, et al, 2006), para esto la alternativa para la multiplicación y obtención de plantas es el uso de las camas enraizadoras, en ellas se estimula la producción de raíces de los cormos, dando posibilidad a que la colonización de los hongos micorrizicos ocurra antes de la siembra definitiva (Coto, 2009).

Debido a los beneficios de la aplicación de micorrizas en las plantas se decidió evaluar las morfoespecies e influencia de los hongos micorrizicos arbusculares sobre el desarrollo y producción de plántulas de plátano barraganete (Musa sp.). 


\section{Metodología}

El trabajo experimental se realizó en la Granja experimental "Rio Suma" de la Universidad Laica "Eloy Alfaro" de Manabí Extensión El Carmen, provincia de Manabí ubicada a $260 \mathrm{msnm}$, la zona está considerada como Trópico Húmedo con temperaturas de $24^{\circ} \mathrm{C}$ en promedio y una precipitación anual promedio de $2.806 \mathrm{~mm}$

El área utilizada del vivero fue de $20 \mathrm{~m} 2$ dividida en tres bloques, uno por repetición, cada bloque fue divido en siete partes, uno por tratamiento, en un total de 21 unidades experimentales; cada unidad experimental contó con 15 cormos libre de plagas y enfermedades, por bloque se utilizaron 105 cormos y en total 315; los sietes tratamientos corresponden a 3 dosis de Hongos Micorrizicos Arbusculares (HMA) en dos tipos de sustratos, más un testigo; se utilizó un diseño completamente al azar (DBCA) en arreglo factorial A x B + N, como factor A los dos tipos de sustratos, el primero fue una mezcla de arena + viruta de balsa y el segundo contó con los mismos materiales más la aplicación de humus; el factor B fueron las tres dosis de HMA 15, 25 y 35\% del peso del cormo, el testigo no presentó humus ni HMA.

Los cormos seleccionados fueron limpiados, se eliminaron todas las raíces y restos de tierra, luego fueron desinfectados con agua caliente $\left(55^{\circ} \mathrm{C}\right)$ durante 20 minutos; después de la limpieza y desinfección, los cormos fueron inoculados en los HMA (Huxtable ${ }^{\circledR}$ Micorriza) de acuerdo a las dosis establecidas para cada uno, luego fueron trasplantados a las camas enraizadoras.

Se evaluaron variables morfológicas como: altura de planta diámetro pseudotallo, numero de hojas, área foliar y porcentaje de supervivencia se realizaron cada semana durante dos meses; además de la efectividad de la micorrización en el sustrato y la raíz, analizadas en el laboratorio de microbiología de ANCUPA ubicado en la ciudad de Santo Domingo; el área foliar se determinó bajo la fórmula: Largo x Ancho x 0,80; los resultados fueron comparados con la prueba de Tukey al 5\% de probabilidad.

\section{Resultados y discusión}

Porcentaje de supervivencia 
La aplicación de Hongos micorrizicos arbusculares y los tipos de sustratos presentaron diferencias significativas $(\mathrm{p}<0,05)$, en el porcentaje de supervivencia de las plántulas de plátano barraganete para la siembra; los tratamientos dispuestos con la aplicación de humus con dosis altas de HMA presentaron la mayor cantidad de plantas efectivas al terminar el desarrollo en el vivero, en promedio superaron el 90\% mientras que el testigo (sin humus y HMA) no superó el 70\% (Figura 1); en condiciones óptimas de manejo de viveros en la multiplicación de plantas de plátano el porcentaje de supervivencia es del 90\% (Coto, 2009), esto indica que la aplicación de HMA y humus en el sustrato, tienen un efecto positivo en el desarrollo de plántulas en vivero, disminuyendo los costos de aplicación de productos químicos; el alto porcentaje de supervivencia de plantas en vivero con aplicación de HMA según Tufiño et al (2011) se debe al incremento en la resistencia de la planta ante ataques de plagas, enfermedades y falta de humedad en el suelo, esto, sumado a la aplicación de humus que mejoran la estructura y características físicas del suelo, además de proporcionar mayor cantidad de nutrientes (Ramos et al, 2016) crean un ambiente óptimo para el desarrollo y supervivencia de las plantas.

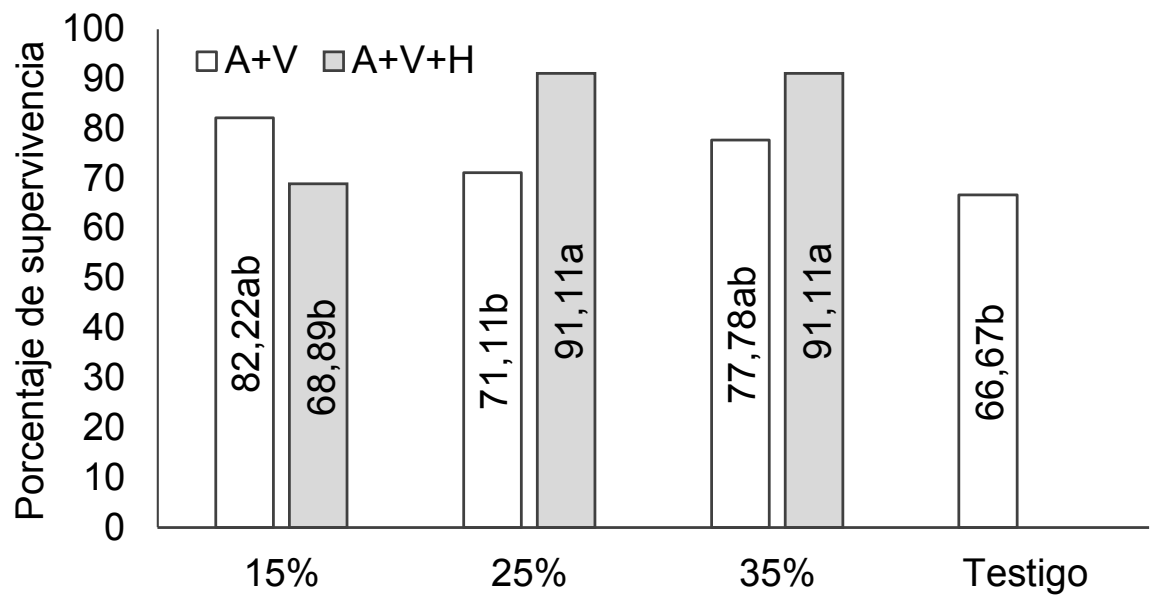

Dosis de HMA

Figura 1. Porcentaje de supervivencia de plántulas de plátano barraganete, en tres dosis de Hongos Micorrizicos Arbusculares y dos tipos de sustratos en El Carmen, Manabí.

Fuente: elaboración propia. 


\section{Hongos micorrizicos arbusculares en el cultivo de plátano en viveros}

\section{Morfología}

El uso de HMA y los tipos de sustratos tuvieron influencia en las variables morfológicas de las plántulas de plátano barraganete, en el análisis estadístico se mostraron diferencias significativas ( $\mathrm{p}<$ $0,05)$ entre los tratamientos para la altura de planta, diámetro de pseudotallo, numero de hojas y área foliar.

González y Cuenca (2008) manifiestan que la inoculación de HMA en musáceas mejoran el desarrollo de las plantas, debido a que facilitan la absorción de nutrientes y mejoran la condición del suelo, Gañán et al (2011) sostienen que la colonización de micorrizas en las raíces a temprana edad de desarrollo incrementa considerablemente el crecimiento vegetativo en viveros; en la altura de planta las tres últimas semanas tuvieron diferencias significativas en la interacción de los factores (Tabla 1), las plantas con dosis de $25 \%$ de HMA en el sustrato con humus terminaron con la mayor altura en promedio, mientras que las plantas bajo la misma dosis en el sustrato sin humus tuvieron los menores promedio en alturas al terminar la fase de vivero, determinando la importancia de la interacción en la aplicación de HMA con humus.

Tabla 1. Respuesta de la inoculación del cormo de plátano barraganete con HMA en la Altura de planta (AP), diámetro de pseudotallo (DP), número de hojas (NH) y área foliar (AF) a los dos meses de establecidos en cama enraizadora con dos tipos de sustratos.

\begin{tabular}{llllll}
\hline Sustrato & HMA & AP & DP & NH & AF \\
\hline \multirow{2}{*}{$\mathrm{A}+\mathrm{V}$} & $15 \%$ & $60,80^{\mathrm{ab}}$ & $3,45^{\mathrm{ab}}$ & $3,53^{\mathrm{b}}$ & $420,32^{\mathrm{abc}}$ \\
& $25 \%$ & $47,20^{\mathrm{b}}$ & $3,12^{\mathrm{b}}$ & $3,27^{\mathrm{bc}}$ & $318,56^{\mathrm{cd}}$ \\
& $35 \%$ & $51,80^{\mathrm{ab}}$ & $3,45^{\mathrm{ab}}$ & $3,67^{\mathrm{ab}}$ & $345,81^{\mathrm{bcd}}$ \\
$\mathrm{A}+\mathrm{V}+\mathrm{H}$ & $15 \%$ & $52,67^{\mathrm{ab}}$ & $3,47^{\mathrm{ab}}$ & $3,53^{\mathrm{b}}$ & $375,47^{\mathrm{bc}}$ \\
& $25 \%$ & $65,20^{\mathrm{a}}$ & $3,69^{\mathrm{a}}$ & $4,07^{\mathrm{ab}}$ & $565,71^{\mathrm{a}}$ \\
\multirow{2}{*}{ Testigo } & $35 \%$ & $60,13^{\mathrm{ab}}$ & $3,87^{\mathrm{a}}$ & $4,47^{\mathrm{a}}$ & $497,76^{\mathrm{ab}}$ \\
\hline
\end{tabular}

Fuente: elaboración propia.

Así como en la altura de planta, el diámetro de pseudotallo también reportó incremento en sus medidas con la inoculación de dosis altas de HMA y la siembra con sustratos con humus; a partir de la quinta semana de establecimiento en vivero los cormos inoculados con 35\% de HMA y en sustrato con humus mantuvieron el mayor diámetro en el pseudotallo, mientras que el testigo sin aplicación de HMA tuvo las medidas más bajas en promedio; este complemento entre las micorrizas y la 


\section{Hongos micorrizicos arbusculares en el cultivo de plátano en viveros}

fertilización orgánica crean un mayor beneficio en el desarrollo morfológico de la planta de plátano, en el trabajo de Villarreal et al (2012) en la aplicación de HMA y la bacteria Azospirillum como biofertilizante se concluyó que las dosis más altas de estos componentes en el banano durante la fase de aclimatación aumenta los valores de altura de planta 7 veces más y el diámetro de pseudotallo 4 veces; el principal motivo de este comportamiento es que las micorrizas al colonizar las raíces le permiten atraer una mayor absorción de nutrientes aunque la difusión de estos sean lentos en el suelo, además de que permitan una mayor captación de humedad (Barea et al, 2008), lo que propicia condiciones ambientales óptimas para el desarrollo de las plantas en viveros; estos resultados eficientes de los HMA según Domínguez et al (2008) se deben a que las plantas colonizadas presentan una mayor absorción de fosforo, lo que consecuentemente incrementa la actividad fotosintética y de transpiración, este aumento de fósforo en el sistema de la planta da paso a una mayor síntesis de proteínas (Navarro y Navarro, 2013).

El número de hojas y el área foliar alcanzada por las plantas fueron superiores con el sustrato con humus, bajo dosis de 35\% de HMA las plántulas lograron producir más de cuatro hojas en promedio al finalizar la fase de vivero, mientras que en dosis de 25\% de HMA la hoja presentó un mayor desarrollo y cobertura, según Orozco-Santos et al (2013) el uso de micorrizas y otros organismos reducen las condiciones favorables para el desarrollo de la sigatoka, principal causante de la disminución foliar, las micorrizas se presentan como un controlador biológico de esta plaga, esto sumado a la introducción de humus en los sustratos mejoran la producción de hojas, Rivera et al (2006) manifiestan que una adecuada relación suelo y humus beneficia en gran medida el área foliar de las plantas.

González y Cuenca (2008) establecieron un ensayo para evaluar el efecto de algunas especies de micorrizas en la producción de hojas en el plátano dominico hartón, encontrando respuestas favorables en la aplicación de Scutellospora heterógama en el cual las plantas alcanzaron el promedio más altos de hojas funcionales; estos resultados se asemejan a los reportados por Villareal et al (2012) en el cual las musáceas responden efectivamente en su biomasa área a la aplicación de HMA; esto debido no solo a concentración de fósforo que produce la colonización de las micorrizas en la raíz, sino a los demás nutrientes como el nitrógeno (Jaizme-Vega y Rodríguez-Romero, 2004), este nutriente absorbido en grandes cantidades por las raíces de la planta, incrementan la producción de biomasa área en el cultivo de plátano, mientras que un nivel bajo de absorción determinará una pobre producción de hojas (Furcal y Barquero, 2014). 


\section{Evaluación de sustratos y raíces}

Los análisis de laboratorio en cuanto al sustrato determinaron que las morfoespecies de micorrizas más encontradas fueron los Glomus y Acaulospora, el primero se halló en todos los sustratos establecidos, mientras que el segundo se encontró solo en las dosis de 15\% y 25\% de HMA inoculados, encontrar hongos tipo Glomus es frecuente en cualquier análisis de sustrato, es el género más común de micorrizas y el más abundante en el suelo (Pérez et al, 2011), los formadores tipo Acaulospora se concentran generalmente en las partes más profundas del suelo (Peña et al, 2007); en la Figura 2 se muestra la relación del número de esporas de cada sustrato en relación al porcentaje de HMA en el que fueron inoculados los colines, en el sustrato sin humus, el número de esporas disminuyó con el aumento de la dosis de HMA, por otro lado en el sustrato con humus la cantidad de esporas encontradas aumentó con el incremento de la dosis de inoculación de HMA, esto determina que bajo una fertilización orgánica las micorrizas presentan un mejor desarrollo en el suelo; Ley-Rivas et al (2015) realizaron un estudio de micorrizas en tomates, los sustratos analizados reportaron diferencias significativas en el número de esporas entre las cepas encontradas del género Glomus, la cual es la más frecuente en la superficie del suelo.

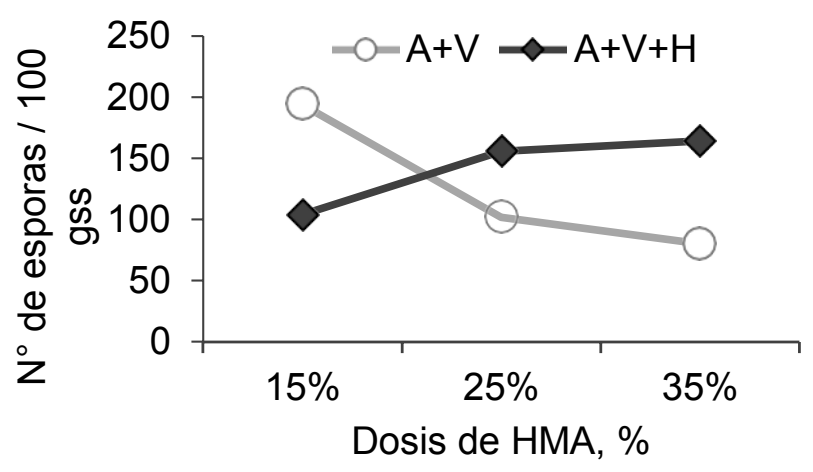

Figura 2. Número de esporas contabilizados en los sustratos con y sin humus, en relación a la dosis de HMA aplicados.

Fuente: elaboración propia.

En la tasa de colonización de micorrizas en las raíces (Figura 3), los resultados de laboratorio presentaron similitudes con los del número de esporas en los sustratos; con la inclusión de humus en la composición del sustrato el porcentaje de colonización incrementa proporcionalmente de acuerdo a la dosis de HMA aplicados, para el sustrato sin humus, el porcentaje de colonización de micorrizas en la raíz alcanzó el valor más alto en la dosis de 25\% de HMA, mientras que en la dosis de 15\% y 


\section{Hongos micorrizicos arbusculares en el cultivo de plátano en viveros}

$35 \%$ el porcentaje disminuye considerablemente; esto debido a la relación con la nutrición del suelo explicada por Rivera et al (2006) en su trabajo de investigación encontró que los valores de colonización de las micorrizas disminuyen de acuerdo al grado de fertilización del suelo, esto indica que el efecto de los HMA en las raíces del plátano no depende de la cantidad inoculada a los colinos, sino de la nutrición del suelo, exceso de nutrientes la eficacia de las micorrizas disminuye.

La capacidad de las plantas para la resistencia a las enfermedades, dependen directamente del porcentaje de colonización en las raíces (González y Cuenca, 2008), mientras más alto el valor de este parámetro mayor resistencia a las enfermedades; para Enríquez y Bernal (2009), la tasa de colonización en las raíces de las micorrizas no depende directamente de la cantidad de HMA inoculada, sino de las condiciones del sustrato; además sostienen que este porcentaje no influye en el desarrollo de la planta.

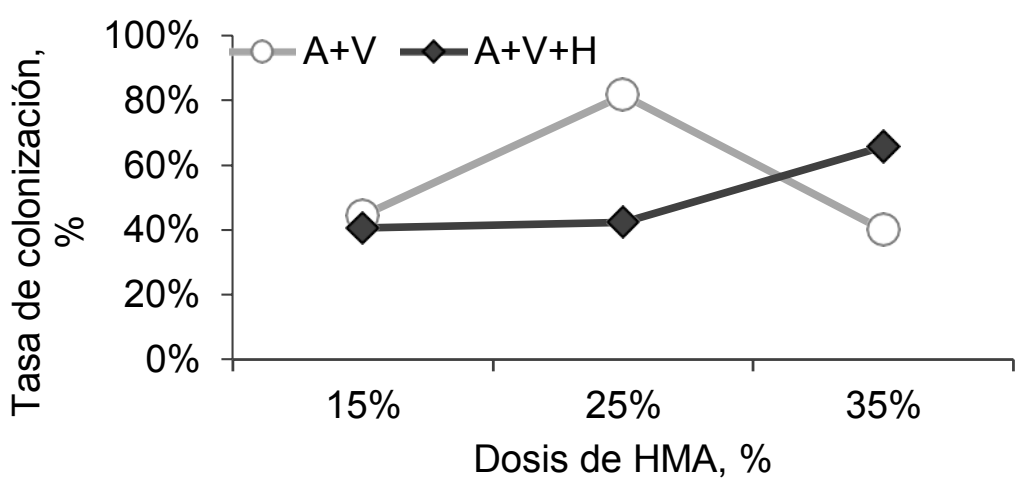

Figura 3. Tasa de colonización de los hongos micorrizicos en las raíces del plátano barraganete en los dos sustratos utilizados y de acuerdo a las dosis aplicadas de HMA.

Fuente: elaboración propia.

En síntesis, la inoculación de colinos de plátano barraganete en HMA y establecidos en sustrato con humus mantiene un alto porcentaje de supervivencia, especialmente en dosis de 25\% y 35\% de HMA. El efecto de los HMA en el desarrollo de las plántulas de plátano en vivero comienza a partir del segundo mes, la dosis de $25 \%$ en sustratos con humus incrementa la altura de las plantas y el área foliar, y la dosis de $35 \%$ en el mismo sustrato mejoran el desarrollo del pseudotallo y la producción de hojas.

En el análisis del sustrato y raíces, la eficiencia de los HMA para el número de esporas y tasa de colonización tienden a mejorar con dosis altas que tengan presencia de humus en el sustrato. 


\section{Referencias Bibliográficas}

Barea, J., Pozo, M., \& Azcón, C. (2018). Significado y aplicación de las micorrizas en agricultura (No. 746) (p. 6). España: Estación Experimental del Zaidín. https://www2.eez.csic.es/mycorrhizaandbioticstresslab/Agricultura-divulgacion\%20micorrizas.pdf

Coto, J. (2009). Guía para multiplicación rápida de cormos de plátano y banano (Segunda). La Lima, Cortés, Honduras: FHIA. http://www.fhia.org.hn/dowloads/proteccion_veg_pdfs/multiplicacion_rapida_de_cormos_de_platan o y banano.pdf

Domínguez, J. A., Planelles, R., Rodríguez, J. A., \& Omeñaca, J. A. S. de. (2008). Influencia de la micorrización con trufa negra (Tuber melanosporum) en el crecimiento, intercambio gaseoso y nutrición mineral de plántulas de Pinus halepensis. Forest Systems, 13(2), 317-327. https://recyt.fecyt.es/index.php/IA/article/view/2376

Enríquez, F. G. E., \& Bernal, G. (2009). Evaluación de la Efectividad de cuatro dosis de Micorrizas Arbusculares bajo cuatro niveles de Fósforo en Vivero de Palmito (Bactris Gasipaes, Hbk), en la zona de Santo Domingo. Eidos, (1), 42-48. https://doi.org/10.29019/eidos.v0i1.40

Franco, J. de D. (2008). Efecto beneficioso de las micorrizas sobre las plantas (p. 27). España: Universidad de Sevilla. http://www.bioscripts.net/col/Apuntes/Nutricion_Vegetal/Trabajo_de_nutricion_vegetal.pdf

Furcal, P., \& Barquero, A. (2014). Fertilización del plátano con nitrógeno y potasio durante el primer ciclo productivo. Agronomía http://www.redalyc.org/resumen.oa?id=43731480005.

Gañán, L., Bolaños-Benavides, M. M., \& Asakawa, N. (2011). Efecto de la micorrización sobre el crecimiento de plántulas de plátano en sustrato con y sin la presencia de nematodos fitoparásitos. Acta Agronómica, 60(4). http://www.redalyc.org/resumen.oa?id=169922450001. 
Hongos micorrizicos arbusculares en el cultivo de plátano en viveros

González, M., \& Cuenca, G. (2008). Respuesta de plantas de plátano (Musa AAB cv. Hartón) a la inoculación con hongos micorrízicos arbusculares nativos e introducidos, bajo condiciones de campo. Revista de la Facultad de Agronomía, 25(3), 470-495. http://www.scielo.org.ve/scielo.php?script=sci_abstract\&pid=S0378$\underline{78182008000300005 \& \ln g=\mathrm{es} \& \mathrm{nrm}=\mathrm{iso} \& \ln \mathrm{l}=\mathrm{es}}$

Guerrero, M. (2011). Guía técnica del cultivo del Plátano. Impreso Múltiples. http://www.centa.gob.sv/docs/guias/frutales/GUIA\%20CULTIVO\%20PLATANO\%202011.pdf INEC. (2016). Encuesta de Superficie y Producción Agropecuaria Continua. http://www.ecuadorencifras.gob.ec/documentos/web-inec/Estadisticas_agropecuarias/espac/espac2016/Presentacion\%20ESPAC\%202016.pdf

Jaizme-Vega, M. C., \& Rodríguez-Romero, A. S. (2004). Uso de micorrizas en banano: Logros y perspectivas. En Publicación Especial (pp. 143-160). Oaxaca, México. http://www.icia.es/icia/index.php?option=com_content\&view=article\&id=2393:uso-de-micorrizasen-banano-logros-y-perspectivas $220 \&$ catid $=126 \&$ Itemid $=257$

Ley-Rivas, J. F., Sánchez, J. A., Ricardo, N. E., \& Collazo, E. (2015). Efecto de cuatro especies de hongos micorrizógenos arbusculares en la producción de frutos de tomate. Agronomía Costarricense, 39(1). [en línea], Disponible en: https://revistas.ucr.ac.cr/index.php/agrocost/article/view/19544

Navarro, G., \& Navarro, S. (2013). Química Agrícola (segunda). México, Mundi-Prensa Libros. Orozco-Santos, M., García-Mariscal, K., Manzo-Sánchez, G., Guzmán, S., Martínez Bolaños, L., Beltrán-García, M. Canto Canche, B. (2013). La Sigatoka negra y su manejo Integrado en banano. Ecuador, Pacifico centro.

Peña-Venegas, C. P., Cardona, G. I., Arguelles, J. H., \& Arcos, A. L. (2007). Micorrizas arbusculares del sur de la Amazonia colombiana y su relación con algunos factores fisicoquímicos y biológicos del suelo. Acta Amazónica, 37(3), 327-336. [en línea], Disponible en: https://oi.org/10.1590/S004459672007000300003 
Pérez, A., Rojas, J., \& Montes, V. D. (2011). Hongos formadores de micorrizas arbusculares: una alternativa biológica para la sostenibilidad de los agroecosistemas de praderas en el Caribe colombiano. Revista Colombiana de Ciencia Animal, 3(2), 366-385. [en línea], Disponible en: https://dialnet.unirioja.es/servlet/articulo?codigo $=3817504$

PROECUADOR. (2015). Análisis Sectorial Plátano. PROECUADOR. http:/www.proecuador.gob.ec/wp-content/uploads/2015/06/PROEC_AS2015_PLATANO1.pdf Ramos, D., Terry, E., Soto, F., Cabrera, A., Martín, G., \& Fernández, L. (2016). Respuesta del cultivo del plátano a diferentes proporciones de suelo y Bocashi, complementadas con fertilizante mineral en etapa de vivero. Cultivos $\quad$ Tropicales, $\quad 37(2), \quad$ 165-174. http://scielo.sld.cu/scielo.php?script=sci abstract\&pid=S0258$\underline{59362016000200020 \& \operatorname{lng}=\mathrm{es} \& \mathrm{nrm}=\mathrm{iso} \& \ln \mathrm{l}=\mathrm{es}}$

Rivera, R., Fernández, F., Hernández, A., Martín, J., \& Fernández, K. (2003). El manejo eficiente de la simbiosis micorrízica, una vía hacia la agricultura sostenible Estudio de caso: el Caribe. Cuba: Ediciones INCA.

Rivera, R., Ruiz, L., Fernández, F., Sánchez, C., Riera, M., Hernández, A., Planas, R. (2006). La Simbiosis micorrízica efectiva y el sistema suelo-planta-fertilizante. Conference paper.

Tufiño, C., Espín, E., Villarreal, T., Proaño, K., \& Medina, M. 2011. Efecto de la interacción de Hongos Micorrícicos Arbusculares (HMA) y la fertilización, sobre el crecimiento y desarrollo de plantas micropropagadas de banano (Musa paradisiaca) durante la fase de aclimatación. Ecuador, Espe. 